\title{
Triagem nutricional em adultos hospitalizados
}

\author{
Nutritional screening in inpatients
}

\author{
Mariur Gomes BEGHETTO ${ }^{1,2}$ \\ Bibiana MANNA ${ }^{3}$ \\ Andréia CANDAL ${ }^{3}$ \\ Elza Daniel de MELLO 2,3,4 \\ Carisi Anne POLANCZYK ${ }^{5,6}$
}

\section{RE S U M O}

Em hospitais, o objetivo de um procedimento de triagem nutricional é identificar indivíduos desnutridos ou em risco de desnutrição, possibilitando intervenção nutricional precoce e melhor alocação de recursos. Diferentes métodos são apresentados na literatura para esta finalidade: Malnutrition Screening Tool, Short Nutritional Assessment Questionnaire, Nutritional Risk Index, Nutrition Risk Score, Nutritional Risk Screening, Mini Nutritional Assessment, Malnutrition Universal Screening Tool, Nutritional Screening Tool, Nutritional Screening Equation. No entanto, o emprego de muitos destes instrumentos está limitado pela inadequada metodologia empregada na derivação e/ou validação, pela seleção de grupos específicos de pacientes, pela pouca praticidade ou por necessidade de um especialista para seu emprego. Na ausência de um padrão de referência para emitir o diagnóstico nutricional, desfechos clínicos relevantes devem balizar a derivação e a validação de novos instrumentos. Este trabalho descreve os instrumentos de triagem nutricional acima referidos e apresenta considerações quanto ao seu emprego para adultos hospitalizados não selecionados.

Termos de indexação: Avaliação nutricional. Desnutrição. Epidemiologia nutricional. Métodos epidemiológicos. Triagem.

\section{A B S T R A C T}

In hospitals, the aim of a nutritional screening procedure is to identify malnourished individuals or those at risk of malnutrition, allowing early nutritional intervention and better allocation of resources. Different methods are presented in the literature for this purpose: Malnutrition Screening Tool, Short Nutritional Assessment

\footnotetext{
${ }^{1}$ Centro Universitário La Salle, Cursos de Graduação em Enfermagem, Nutrição e Fisioterapia. Av. Victor Barreto, 2288, Centro, 92010-000, Canoas, RS, Brasil. Correspondência para/Correspondence to: M.G. BEGHETTO. E-mail: $<$ mbeghetto@hcpa.ufrgs.br>.

2 Hospital de Clínicas de Porto Alegre, Comissão de Suporte Nutricional. Porto Alegre, RS, Brasil.

3 Hospital de Clínicas de Porto Alegre, Serviço de Nutrologia. Porto Alegre, RS, Brasil.

${ }^{4}$ Universidade Federal do Rio Grande do Sul, Departamento de Pediatria. Porto Alegre, RS, Brasil.

5 Universidade Federal do Rio Grande do Sul, Departamento de Medicina Interna, Programas de Pós-Graduação em Cardiologia e Epidemiologia. Porto Alegre, RS, Brasil.

${ }^{6}$ Hospital de Clinicas de Porto Alegre, Serviço de Cardiologia. Porto Alegre, RS, Brasil.
} 
Questionnaire, Nutritional Risk Index, Nutrition Risk Score, Nutritional Risk Screening, Mini Nutritional Assessment, Malnutrition Universal Screening Tool, Nutritional Screening Tool, Nutritional Screening Equation. However, the use of many of these instruments is limited by inadequate methodology employed in their derivation and / or validation, by selecting specific groups of patients, by their limited feasibility or by requiring an expert to apply it. In the absence of a reference standard to deliver the nutritional diagnosis, relevant clinical outcomes must delimitate the derivation and validation of new instruments. This paper describes the above mentioned nutritional screening instruments and presents considerations regarding their use in non-selected adult inpatients.

Indexing terms: Nutritional assessment. Malnutrition. Nutritional epidemiology. Epidemiologic methods. Screening.

\section{N T R O D U ÇÃ O}

A manutenção do estado nutricional é importante para a preservação e a recuperação da saúde. Ainda assim, a desnutrição hospitalar é um evento prevalente na atualidade ${ }^{1,2}$, contribuindo para o aumento da morbimortalidade ${ }^{3-5}$, tempo ${ }^{2,5}$ e do custo com a hospitalização ${ }^{6,7}$, e para a piora na qualidade de vida ${ }^{8}$. Diferentes fatores colaboram para este desfecho em adultos hospitalizados: ação da doença de base e de co-morbidades, ingestão insuficiente, efeitos colaterais de medicamentos, inatividade física ${ }^{3,9}$ e desconsideração da importância dos cuidados nutricionais pelos profissionais de saúde 5,10,11. A identificação precoce desses fatores possibilita a adoção de melhor manejo nutricional.

A avaliação nutricional envolve variáveis subjetivas e objetivas ${ }^{12}$, examinadas detalhadamente por profissionais experientes. Portanto, 0 diagnóstico nutricional demanda tempo e outros recursos. Por outro lado, a triagem nutricional é o procedimento que busca identificar indivíduos desnutridos ou em risco de desnutrição, com o propósito de verificar se uma avaliação nutricional adicional, mais detalhada, é necessária ${ }^{13}$. A triagem nutricional identifica fatores que, se presentes, colocam o paciente em risco de desnutrição e de apresentar complicações relacionadas a ela ${ }^{14}$. Dessa forma, a triagem nutricional sinaliza, precocemente, pacientes que poderiam beneficiarse de terapia nutricional. Nesse sentido, deve ser um procedimento rápido, executado pela equipe de saúde que realiza a admissão hospitalar, buscando identificar se o paciente: (a) não é de risco, mas deve ser reavaliado em intervalos regulares, (b) é de risco e necessita seguir um plano nutricional; (c) é de risco, mas problemas metabólicos e funcionais impedem a adoção de um protocolo padronizado, ou se (d) há dúvida se o paciente é de risco. Nas duas últimas condições, há necessidade de referenciar o paciente para uma avaliação mais detalhada, por um especialista na área ${ }^{12}$. Diferentes instrumentos têm sido propostos para avaliar o risco nutricional. No entanto, o emprego de parte desses instrumentos apresenta limitações como: (a) falta de validação, (b) uso exclusivo de critérios subjetivos, (c) inclusão de pacientes selecionados, (d) pouca praticidade e (e) necessidade de avaliação por nutricionista. Nesse sentido, esta comunicação se propõe a descrever diferentes instrumentos de triagem nutricional disponíveis e apresentar considerações quanto ao seu emprego para adultos hospitalizados não selecionados.

\section{MÉTO D OS}

Entre dezembro de 2005 e julho de 2006 foi realizada uma revisão de literatura, para identificar artigos originais de validação de métodos de triagem nutricional na base de dados do Medline, utilizando-se as palavras-chave: \#1: nutritional screening; \#2: nutritional assessment; \#3: risk score; \#4: method; \#5: hospital inpatient; \#6: validation; \#7: accuracy; \#8: reproducibility; \#9: predict; \#10: clinical outcome. Não foi utilizado filtro para a busca de data. Referências citadas em estudos encontrados na busca e em livros texto específicos de avaliação nutricional, também foram avaliadas. Quando necessário, foram feitos 
contatos com os autores para aquisição de algumas publicações. Foram avaliados os artigos originais que descreveram métodos de triagem nutricional quanto ao seu objetivo inicial, ao procedimento de derivação e a validação e aos principais resultados.

\section{Métodos de Triagem Nutricional}

\section{Malnutrition Screening Tool}

O Malnutrition Screening Tool (MST) $)^{15}$ originou-se de um questionário derivado da revisão da literatura e da experiência dos autores. Foi aplicado em 408 adultos internados em diferentes especialidades de clínica e cirurgia de um hospital australiano, especialmente em pacientes das áreas de ginecologia e cirurgia plástica $(34,6 \%$ da amostra). Os autores atribuíram valores entre 0 e 5 para as possíveis respostas categorizadas às 21 questões que versavam sobre: perda de peso, apetite e morbidade. A Avaliação Nutricional Subjetiva Global (ANSG), descrita por Detsky et al. (categorias B e C), foi adotada como padrãoouro para o diagnóstico de desnutrição ${ }^{15}$. A ANSG e o questionário elaborado foram aplicados nas primeiras $48 \mathrm{~h}$ da admissão e os pacientes tiveram seu Índice de Massa Corporal (IMC) calculado a partir do peso e altura corporal referidas. Prega tricipital e circunferência muscular do braço foram

Tabela 1. Formato final do Malnutrition Screening Tool.

\begin{tabular}{lc}
\hline Questões & Pontuação \\
\hline Você teve perda recente de peso? & 0 \\
Não & 2 \\
Não sabe & \\
Se sim, de quanto (em kg) foi a sua perda de peso? & 1 \\
1-5 & 2 \\
6-10 & 3 \\
$11-15$ & 4 \\
$>15$ & \\
Você está comendo menos por redução do apetite? & 0 \\
Não & 1 \\
Sim & $\mathbf{1 3}$ \\
\hline Total & \\
\hline
\end{tabular}

aferidos. Resultados de testes laboratoriais (proteínas totais, albumina, pré-albumina, contagem de linfócitos e de leucócitos totais e proteína-C reativa) foram revisados nos prontuários. Várias combinações das 21 questões foram testadas. As questões identificadas como de maior sensibilidade e especificidade ("Você tem se alimentado menos por apresentar diminuição do apetite?" e "Você apresentou perda de peso não intencional recentemente?") passaram a constituir o Malnutritional Screening Tool (MST) (Tabela 1), embora também integrassem a ANSG. Para cada questão do MST foram atribuídos pontos que, quando somados, constituíam um escore. Os valores de escore $\geq 2$ foram escolhidos como ponto de corte para identificação de risco nutricional, por apresentarem sensibilidade e especificidade de $93 \%$ em detectar desnutrição (categorias B e ( da ANSG).

Posteriormente, os parâmetros objetivos de avaliação do estado nutricional foram comparados nos grupos de pacientes classificados em risco nutricional (MST=2 a 5) ou sem risco de desnutrição (MST=0 a 1). Foram identificadas diferenças estatisticamente significantes entre os grupos, com exceção dos valores de linfócitos e leucócitos totais. Em uma amostra de 32 pacientes, a reprodutibilidade do instrumento foi testada por 3 diferentes duplas de nutricionistas. A menor concordância obtida entre as duplas foi de $93 \%$ (kappa=0,84) e a maior foi de 97\% (kappa=0,93). Os autores concluem que o MST é um instrumento fácil, simples, reprodutível e válido, que pode ser empregado por qualquer profissional de saúde, por familiar ou pelo próprio paciente na admissão hospitalar. Cabe considerar que o instrumento de ANSG, adotado como padrão de referência, contém ambas as questões incluídas no MST. Sendo assim, seria esperado que estes fossem itens identificados como de elevada sensibilidade e especificidade, quando comparados à ANSG .

\section{Short Nutritional Assessment Questionnaire}

OShort Nutritional Assessment Questionnaire $(\mathrm{SNAQ} \odot)^{16}$ foi desenvolvido e validado no intuito 
de encontrar um mecanismo de triagem do estado nutricional que fosse válido e de rápida aplicação. Para tanto, foram avaliados 588 adultos internados em diferentes especialidades de clínica, cirurgia e oncologia do VU University Medical Center de Amsterdam. Na $1^{\text {a }}$ etapa, à admissão hospitalar, 291 pacientes foram pesados, informaram sua altura corporal e responderam a 26 questões que abrangiam sintomas e fatores de risco para desnutrição, provenientes de questionários de qualidade de vida (EORTC-C30 e EORTC H\&N 35) e de outros instrumentos de avaliação nutricional (Nutricia Nutritional Screening List, Mini Nutritional Assessment, Subjective Global Assessment), além de outras questões sugeridas por nutricionistas. Foram adotados os seguintes critérios para a classificação dos pacientes: (1) gravemente desnutridos: IMC $<18,5 \mathrm{~kg} / \mathrm{m}^{2}$ ou perda de peso não intencional acima de $5 \%$ no último mês ou acima de $10 \%$ nos últimos 6 meses, (2) moderadamente desnutridos: perda de peso entre $5 \%$ e $10 \%$ em 6 meses e IMC $>18,5 \mathrm{~kg} / \mathrm{m}^{2}$ e (3) bem nutridos: perda de peso abaixo de $5 \%$ nos últimos 6 meses e IMC $>18,5 \mathrm{~kg} / \mathrm{m}^{2}$. Inicialmente, para seleção das questões mais preditivas de desnutrição, foi realizada regressão logística binomial. A presença de desnutrição (moderada ou grave) serviu como padrão de referência. A seguir, foi procedida modelagem polinomial, a partir da qual as 3 categorias já descritas foram adotadas como padrão de referência. O escore SNAQ@ foi, então, obtido utilizando-se os valores dos coeficientes de regressão encontrados, tendo sido estabelecidos seus parâmetros de classificação a partir dos melhores pontos entre sensibilidade e especificidade. Das 26 questões iniciais, 4 foram mantidas no escore final: "Você perdeu mais que 6kg em 6 meses?", atribuído 3 pontos, (Odds Ratio - OR: 267,0; IC95\%: 30,0-2376,2), "Você perdeu mais que $3 \mathrm{~kg}$ em 6 meses?", atribuído 2 pontos, (OR: 37,7; IC95\%: 12,5-113,6), "Você está reduzindo seu apetite há 1 mês ou mais?", atribuído 1 ponto, (OR: 4,2; IC95\%: 1,5-11,4) e "Você está utilizando suplementos por via oral ou enteral no último mês?", atribuído 1 ponto, (OR: 4,3; IC95\%: 1,4-19,9).
A coorte de validação foi constituída por outros 297 pacientes, avaliados por enfermeiras na admissão hospitalar, utilizando o escore SNAQ@ O. Os pacientes foram classificados como eutróficos (SNAQ $\odot<2$ pontos), moderadamente desnutridos (SNAQ@ $=2$ pontos) ou gravemente desnutridos (SNAQ@ $\geq 3$ pontos). Aqueles classificados como moderadamente desnutridos recebiam suplementação energético-protéica e os gravemente desnutridos recebiam, também, atendimento pelo nutricionista. O encaminhamento ao nutricionista foi qualificado, de forma subjetiva, pelo nutricionista que realizou o atendimento como: "muito necessário", "moderadamente necessário" ou "desnecessário". A acurácia e a capacidade do SNAQ@ em predizer desnutrição, conforme os critérios estabelecidos na $1^{\text {a }}$ etapa, também foram avaliadas. A concordância entre enfermeira e enfermeira (kappa - $\kappa=0,69$; IC95\%\%: 0,45 - 0,94) e entre enfermeira e nutricionista ( $\kappa=0,91$; IC95\% \%: 0,8-1,03) foi verificada pela avaliação em duplicata de 2 grupos de 47 pacientes. A área sob a Receive Operator Caracteristic Curve (curva ROC), encontrada nas classificações "moderadamente desnutrido" (SNAQ@ $\geq 2$ ) e "gravemente desnutrido" (SNAQ@ $\geq 3$ ), foi de $0,85(0,79-0,9)$. O SNAQ@ é um escore validado em todas as suas etapas, no qual a perda de peso, de apetite e o uso de suplementos alimentares foram as variáveis mantidas na equação encontrada. No entanto, os parâmetros adotados como padrão de referência na validação podem apresentar alta colinearidade com as variáveis finais do modelo, já que tanto o percentual de perda de peso quanto o IMC referem-se ao peso corporal dos indivíduos, e estas foram as questões de maior impacto no escore final. Assim, as altas sensibilidade e especificidade parecem ser esperadas, já que a perda de peso é contemplada tanto como variável independente, quanto dependente. Também, cabe observar que mesmo sem ajuste do modelo final para variáveis clínicas e demográficas, os valores dos intervalos de confiança dos OR do modelo final foram de grande variabilidade. 


\section{Nutritional Risk Index}

Pelo menos 2 escores denominados Nutritional Risk Index (NRI) estão disponibilizados na literatura, ambos da década de 8017,18.

O Nutritional Risk Index foi desenvolvido na década de 80 , a partir de 16 questões derivadas do National Health and Nutritional Examination Survey (NHANES I), que avaliavam restrição dietética, mudanças nos hábitos alimentares, fatores e morbidades que afetam a ingestão. O objetivo foi desenvolver um escore de identificação de idosos em risco de desenvolver condições clínicas relacionados ao seu estado nutricional que levassem à elevada utilização de Serviços de Saúde (consultas médicas em emergências e hospitais). Visava identificar pessoas que se beneficiariam de avaliação diagnóstica adicional e de intervenções nutricionais, de forma preventiva. Em 1982, um primeiro estudo de base populacional avaliou 401 idosos (idade $\geq 65$ anos) não hospitalizados, americanos, em 3 momentos distintos: inclusão (T1), após 4 meses (T2) e após 12 meses (T3), quanto a: percepção sobre sua saúde, atividades de vida diária, auto-estima, utilização de serviços de saúde, dados demográficos e medidas antropométricas. Os sujeitos também responderam a 16 questões derivadas do NHANES I que contemplavam: restrição alimentar em decorrência de limitação clínica, problemas de mastigação, deglutição e dentição, sintomas do trato gastrintestinal, perda de peso, cirurgia abdominal, anemia, fumo, uso de medicamentos, dieta ${ }^{17}$. Os autores buscavam testar o instrumento quanto à sua consistência e reprodutibilidade, comparando as freqüências de respostas nos 3 diferentes tempos de avaliação. Para cada resposta afirmativa deveria ser somado 1 ponto. Os pacientes foram classificados como baixo risco, quando o escore foi $<4$ pontos, risco moderado, quando o escore esteve entre 5 a 7 pontos e alto risco, quando o escore foi $\geq 8$. Os autores concluíram que o NRI é um instrumento reprodutível e válido para predizer a utilização de serviços de saúde por idosos não hospitalizados, ainda que apresente algumas limitações. A seguir, outro estudo foi conduzido para avaliar se o NRI poderia substituir métodos de avaliação nutricional mais complexos e $\operatorname{caros}^{19}$. Nessa ocasião, foram incluídos 377 homens não hospitalizados, com idade $\geq 55$ anos, predominantemente brancos, ao consultarem clínicos ou geriatras, do Veterans Administration Medical Center. Os pacientes foram avaliados por meio de antropometria, exames laboratoriais, recordatório alimentar, dados demográficos, hábitos de vida, utilização de serviço de saúde, padrão psicossocial e capacidade funcional. Indivíduos com IMC $<20 \mathrm{~kg} / \mathrm{m}^{2}$ foram classificados como muito magros e os com IMC $>30 \mathrm{~kg} / \mathrm{m}^{2}$, como obesos. Valores laboratoriais alterados (hemoglobina $<14 \mathrm{mg} / \mathrm{dL}$, hematócrito $<42 \%$, contagem total de linfócitos $<1.000$ células/ $\mu \mathrm{L}$ e albumina sérica $<3,5 \mathrm{mg} / \mathrm{dL}$ ) geraram 1 ponto para cada resultado, assim como o uso de medicamentos que interferissem no estado nutricional. Os resultados obtidos foram semelhantes aos do estudo anterior, sendo encontrada uma consistência interna modesta e concordância interobservadores de 0,69 . Os autores apontam limitações importantes à generalização de seus achados como: amostra composta exclusivamente por indivíduos do sexo masculino e usuários do sistema para veteranos, poucos não brancos, e dificuldades com a confiabilidade, dada a utilização de questionário auto-aplicável.

\section{Nutrition Risk Index}

O estudo desenvolvido por Buzby et al. ${ }^{18}$ objetivou avaliar a freqüência de complicações e morte perioperatória em cirurgias abdominais e torácicas, não cardíacas, e definir marcadores de desnutrição que identificassem pacientes elegíveis para terapia nutricional parenteral. Foram avaliados 368 pacientes do Veterans Affairs Medical Center após laparotomia ou toracotomia eletiva, sendo excluídos os pacientes com expectativa de sobrevida $<90$ dias, os que receberam nutrição parenteral (NP) nos 15 dias anteriores à inclusão, ou os que foram submetidos a qualquer cirurgia nos 30 dias anteriores. Na admissão, os pacientes 
foram submetidos à avaliação nutricional laboratorial (albumina e pré-albumina) e antropométrica (peso corporal, percentual de perda de peso em relação ao peso usual, percentual do peso ideal, prega cutânea tricipital e circunferência muscular do braço). Ao longo da internação foram acompanhados para a ocorrência de complicações e evolução para óbito. Pacientes foram considerados desnutridos quando apresentaram pelo menos 2 marcadores do estado nutricional alterados. Todas as combinações dos 7 marcadores do estado nutricional foram avaliadas quanto a: sensibilidade, especificidade e valor preditivo, sendo que 7 destas combinações apresentaram melhor desempenho preditor (sensibilidade entre $54,0 \%$ e $61,3 \%$ e especificidade entre $66,8 \%$ e $71,1 \%$ ). Para a escolha de quais métodos de avaliação do estado nutricional deveriam ser incorporados ao escore, foi levado em consideração que a determinação de albumina e pré-albumina é de mais fácil padronização e monitoramento do que a aferição da Prega Cutânea Tricipital (PCT) e da Circunferência Muscular do Braço (CMB). Além disso, que só haveria necessidade de avaliar o peso corporal por meio de uma das 3 variáveis: peso, percentual de perda de peso em relação ao peso usual ou percentual do peso ideal. Após ajustes, a equação preditora de desfechos clínicos (morte, sobrevivência com complicações ou sobrevivência livre de complicações) foi chamada de Nutritional Risk Index $[\mathrm{NRI}=(1,519 \times$ albumina sérica $\mathrm{g} / \mathrm{L})+41,7$ $\times$ (peso atual/peso usual)]. Os pacientes foram classificados de acordo com o valor do NRI obtido: (a) ausência de risco: NRI>100, (b): baixo risco: $\mathrm{NRI}=97,5$ a 100 e (c) risco moderado: $\mathrm{NRI}=83,5 \mathrm{a}$ 97,5. Desde então, este índice vem sendo utilizado para avaliar indivíduos em diferentes condições e desfechos clínicos ${ }^{20,21}$ e acompanhar o impacto de intervenções nutricionais ${ }^{22}$.

\section{Nutrition Risk Score}

O Nutrition Risk Score (NRS) ${ }^{23}$ foi elaborado a partir de outros métodos utilizados: (1) perda de peso (percentual e tempo), (2) IMC para adul- tos, percentil de peso para a altura para crianças, (3) ingestão alimentar (apetite, capacidade de alimentação e absorção) e (4) fatores de estresse (condições clínicas e necessidade de terapia nutricional). Cada uma dessas informações foi pontuada com valores entre 0 e 3 , arbitrados pelos autores. O escore foi desenhado para refletir o estado de desnutrição, devendo ser de fácil utilização, reprodutível e aplicável em todos os pacientes adultos e pediátricos, em 10 diferentes especialidades clínicas e cirúrgicas. Na etapa de estudo piloto, 20 pacientes, aleatoriamente selecionados, foram avaliados em duplicata por 10 nutricionistas. Cada paciente teve o valor de NRS calculado e correlacionado ao valor do Nutritional Risk Inde ${ }^{24}$ e à impressão clínica do nutricionista (correlação de Spearman). Os pacientes foram classificados em 3 categorias de risco nutricional: (1) baixo, (2) médio e (3) alto, não tendo sido apresentados os pontos de corte para a categorização. No estudo piloto, nas 40 avaliações efetivadas, foi encontrada correlação intermediária entre NRS e NRI $(r=0,68 ; p<0,001)$ e forte com a impressão dos nutricionistas $(r=0,83$; $p<0,001)$. Em uma segunda etapa, 153 pacientes hospitalizados foram avaliados por 12 nutricionistas, respeitando a distribuição de internações por especialidade do ano anterior. O objetivo foi identificar a ação adotada com os pacientes: referência para terapia nutricional, monitoramento, ou nenhuma ação. Para verificar a correlação do NRS quando empregado por enfermeiras, 19 pacientes foram avaliados, também, por estas profissionais. A correlação entre nutricionistas e enfermeiras foi de $0,80(p<0,001)$, mas para todos os 19 pacientes, o escore das enfermeiras excedeu ao das nutricionistas. Quase a totalidade dos profissionais julgou que o NRS é de fácil aplicação, atingindo um dos objetivos iniciais dos autores. Mais recentemente, Corish et al..$^{25}$ identificaram que tanto o Nutrition Risk Index ${ }^{22}$, quanto o Nutrition Risk Score ${ }^{23}$, falham em identificar desnutrição (IMC $<20 \mathrm{~kg} / \mathrm{m}^{2}$ e prega tricipital ou circunferência muscular do braço <percentil 15) em muitos dos 359 pacientes avaliados. 


\section{Nutritional Risk Screening ${ }^{6}$}

Este mecanismo de triagem nutricional foi desenvolvido a partir do conceito de que a terapia nutricional é indicada para pacientes desnutridos e/ou criticamente doentes, nos quais o requerimento nutricional está aumentado ${ }^{26}$. Assim, um grupo de pesquisadores dinamarqueses realizou uma análise retrospectiva de estudos desenhados para avaliar o benefício clínico da terapia nutricional, no qual os pacientes tiveram o estado de desnutrição e gravidade clínica categorizada em: (a) ausente, (b) leve, (c) moderada e (d) grave. Utilizando uma pontuação variável entre os valores 0 e 6, os pacientes foram classificados como em risco, quando obtinham somatório $\geq 3$ pontos. A desnutrição foi diagnosticada a partir de variáveis mais freqüentemente utilizadas em instrumentos de triagem: IMC, percentual de perda de peso, mudança na aceitação da via oral e seu impacto clínico (Quadro 1).

Para o cálculo final do escore, os pontos obtidos na categoria relacionada ao estado nutricional deviam ser somados aos da gravidade da doença. Quando o paciente apresentou idade $\geq 70$ anos, foi adicionado um ponto ao escore, para efetuar uma correção para a idade. Pacientes com escore ajustado para a idade $\geq 3$ tiveram recomendação de início de terapia nutricional. A análise da razão de probabilidade para efeito positivo da intervenção nutricional, em relação a diferentes pontos de corte do escore, mostrou que, com o aumento do escore, houve maior benefício tanto da terapia nutricional enteral, quanto parenteral, ainda que com muita variabilidade na precisão das estimativas.

\section{Mini Nutritional Assessment}

A derivação e a validação do Mini Nutritional Assessment (MNA) foi realizada em parceria entre Toulouse University Hospital (França), University of New Mexico (EUA) e Nestle Research Center (Switzerland), em uma seqüência de dois estudos $^{27,28}$. No primeiro deles, foi procedida a derivação do MNA, a partir de questões englobando medidas antropométricas (peso, altura e perda de peso), avaliação global (estilo de vida, uso de medicamentos e mobilidade), dieta (número de refeições, ingestão de alimentos, hidratação e autonomia para alimentar-se) e avaliação subjetiva (autopercepção do estado nutricional e saúde), quando foram avaliados 105 idosos hospitalizados e 50 idosos participantes de atividades de Universidade para Terceira Idade. Foram consi-

Quadro 1. Critérios para classificação nutricional estabelecidos no Nutritional Risk Screening ${ }^{26}$

\begin{tabular}{lll}
\hline & Deterioração do estado nutricional & Gravidade da doença (grau de estresse) \\
\hline 0: Ausente & Estado nutricional normal & Requerimento nutricional normal. \\
1: Leve & Perda de peso $>5 \%$ em 3 meses & Fratura quadril, pacientes crônicos (especialmente \\
& OU & cirróticos), doença pulmonar obstrutiva crônica, pa- \\
& Aceitação da via oral entre 50 e $75 \%$ da estimativa de & cientes em hemodiálise, diabéticos e oncológicos. \\
& requerimento há 1 semana & \\
2: Moderado & Perda de peso $>5 \%$ em 2 meses & Cirurgia abdominal de grande porte, acidente vas- \\
& OU & cular cerebral, pneumonia grave, leucemia. \\
& IMC $=18,5$ a 20,5 + piora das condições gerais & \\
& OU & \\
& Aceitação da via oral entre $25 \%$ e $50 \%$ da estimativa de & \\
requerimento há 1 semana & \\
& Perda de peso $>5 \%$ em 1 mês $(\approx 15 \%$ em 3 meses) & Traumatismo craniano, transplante de medula ós- \\
& OU & sea, pacientes críticos (Acute Physiology and Chronic \\
& IMC $<18,5+$ piora das condições gerais & Health Evaluation - APACHE $\geq 10)$.
\end{tabular}


derados nutridos os idosos com MNA $\geq 24$, em risco nutricional, aqueles com MNA entre 17 e 23,9 e desnutridos, os com MNA <17. Todos os idosos também foram submetidos à avaliação nutricional completa: antropometria (IMC, circunferência braquial, prega cutânea tricipital, circunferência muscular do braço, prega subscapular), bioquímica (albumina, transferrina, pré-albumina, proteínas ligadas ao retinol, ceruloplasmina, proteína-C reativa, glicoproteína alfa-1, coleterol, vitaminas $A, D, E, B_{1}, B_{6}, B_{12}$ e folato, cobre e zinco) hematológica, dietética (recordatório de 3 dias combinado com inquérito de freqüência alimentar) e funcional geriátrica (Mini-Mental, Activities of Daily Living). Em comparação com o padrão de referência (avaliação nutricional completa), somente $2,2 \%$ dos idosos foram mal classificados e o diagnóstico de desnutrição pelo MNA (MNA <17) mostrou-se sensível, específico e preditivo ( $S=96 \%, E=98 \%$ e $V P=97 \%$ ). Em um segundo estudo, 120 idosos doentes e 30 saudáveis foram avaliados pelo MNA, antropometria e marcadores bioquímicos para a etapa de validação do escore. Foi encontrada concordância em 78\% das classificações dos idosos (categorias: nutrido e desnutrido). Dentre os demais idosos, nenhum deles foi classificado como nutrido pelo padrão de referência e em risco ou desnutrido pelo MNA. Estudos em outros grupos de pacientes, comparando o MNA a diferentes parâmetros de avaliação do estado nutricional ou a desfechos clínicos ${ }^{29}$, têm sido desenvolvidos.

\section{Malnutrition Universal Screening Tool}

O Malnutrition Universal Screening Tool (MUST) foi desenvolvido pelo Malnutrition Advisory Group of the British Association for Parenteral and Enteral Nutrition com apoio de entidades de diferentes profissionais de saúde, como proposta de método de triagem nutricional para adultos de todos os tipos de internação ${ }^{30}$. Tendo em vista a ausência de padrão de referência para o diagnóstico de desnutrição e risco nutricional, os autores adotaram três critérios que refletissem a evolução do paciente: (1) perda não intencional de peso (passado), (2) IMC (presente) e (3) efeito da doença aguda sobre a ingestão alimentar (futuro) (Figura 1).

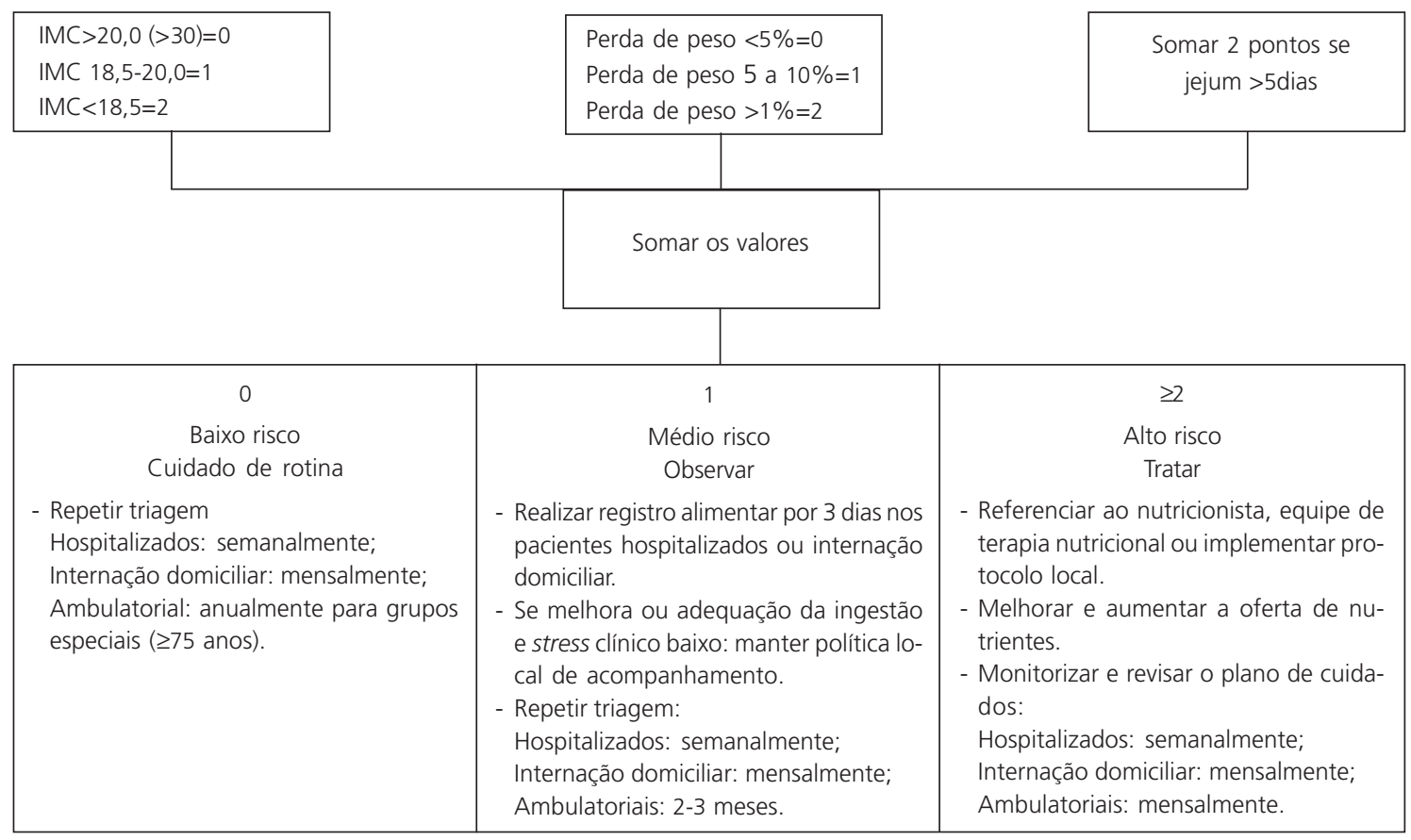

Figura 1. Malnutrition Universal Screening Too/30. 
Os pacientes foram agrupados em três categorias: baixo, médio e alto risco de desnutrição. Posteriormente, as duas últimas categorias foram unificadas. Utilizando duas categorias para classificar os pacientes quanto ao seu risco nutricional (em risco ou livre de risco), a concordância entre o MUST e outros 7 instrumentos já disponíveis foi comparada: Merec e Hickson \& Hill citados por MUST ${ }^{30}$ (pacientes de gastroenterologia não hospitalizados, $n=50$ ), Nutritional Risk Score e Malnutritional Screening Tool (pacientes clínicos hospitalizados, n=75), MNA-SF (pacientes idosos clínicos e cirúrgicos hospitalizados, $n=86$ ), Avaliação Nutricional Subjetiva Global (pacientes clínicos hospitalizados e com idade $<65$ anos, $n=50$ ) e Undernutritional Risk Score (pacientes de cirurgia geral hospitalizados, $n=52$ ). A maior concordância foi encontrada entre MUST e Merec ( $\kappa=0,893$ desviopadrão - $\mathrm{DP}=0,077$ ) e a menor, entre MUST e Undernutritional Risk Score $(\kappa=0,255 \mathrm{DP}=0,101)^{30}$. Em todas as comparações com os demais métodos, o MUST foi adotado como padrão de referência. Além disso, IMC, perda de peso e ingestão alimentar são, isoladamente ou em conjunto, componentes dos 7 demais métodos avaliados.

\section{Nutritional Screening Tool}

Três escores denominados Nutritional Screening Tool (NST) estão descritos a seguir.

Laporte et al. ${ }^{31}$, com a finalidade de desenvolver um instrumento simples, sensível, específico e com valores preditivos $\geq 80 \%$ para detectar desnutrição em pacientes adultos e idosos, constituíram o Nutritional Screening Tool. Foram avaliados 111 pacientes agudamente doentes do Campbellton Regional Hospital (54 adultos e 57 idosos) e 49 idosos cronicamente doentes do Village Campbellton Nursing Home, Canadá. Seguindo a recomendação da American Dietetic Association, 9 variáveis relacionadas a antropometria, bioquímica, dietética, clínica e estado funcional foram escolhidas para avaliar o risco de desnutrição e pontuadas de 1 (desnutrição leve) a 3 (desnutrição grave). A partir do somatório dos pontos obtidos em cada uma das 9 questões, o paciente foi classificado como sem risco $(0$ a 2 pontos), risco leve ( 3 a 5 pontos), risco moderado ( 6 a 8 pontos) ou alto risco para desnutrição $(\geq 9$ pontos).

Os pacientes hospitalizados foram avaliados por um técnico em nutrição, em até 72 horas da admissão, e os demais tinham o peso mensalmente verificado. Duas nutricionistas realizaram avaliações nutricionais completas, após $24 \mathrm{~h}$ da avaliação de triagem. A partir da presença de 4 parâmetros anormais (indicadores antropométricos, bioquímicos ou dietéticos), os pacientes foram classificados como: (1) nutridos, (2) levemente desnutridos, (3) moderadamente desnutridos ou (4) gravemente desnutridos. Este diagnóstico nutricional foi adotado como padrão de referência para a validação do escore. Condições clínicas, albumina, percentagem de perda de peso e IMC foram as variáveis significativamente associadas, de modo independente, à predição de desnutrição (diagnóstico das nutricionistas). Visto que a obtenção de histórico clínico é demorada, esta variável foi excluída da modelagem. Assim, o modelo constituiu-se das variáveis IMC, albumina e perda de peso, sendo o escore 3 identificado como melhor ponto de corte para predizer desnutrição. Considerando que albumina não é possível de ser coletada em muitos dos indivíduos e que em outros não é possível obter dados confiáveis sobre a história de perda de peso, modelos mantendo somente IMC e uma dessas variáveis foram avaliados, sendo o escore 2 o melhor ponto de corte para predizer desnutrição. Para adultos hospitalizados, os modelos que incluíram IMC e percentual de perda de peso (modelo 1) ou IMC e albumina (modelo 2) mostraramse específicos $(81,3 \%$ e $97,9 \%$ ) e de elevado valor preditivo $(75,9 \%$ e $90,7 \%)$, mas não sensíveis (33,3\%, em ambos os modelos). Para idosos hospitalizados, os modelos 1 e 2 mostraram-se sensíveis (78,6\% e 92,9\%), específicos (76,7\% e $83,7 \%)$ e de elevado valor preditivo $(77,2 \%$ e $86,0)$, enquanto para idosos não hospitalizados o modelo 1 mostrou-se sensível, específico e 
preditivo $(85,5 \%, 75,9 \%$ e $79,6 \%$, respectivamente). Já o modelo 2, mostrou-se sensível $(90,0 \%)$, mas pouco específico e preditivo $(44,8 \%$ e $63,3 \%$ ). Não se deve deixar de considerar que as variáveis incluídas no modelo são as mesmas utilizadas pelas nutricionistas para a emissão do diagnóstico nutricional de referência, o que pode superestimar a acurácia do teste.

A fim de testar a concordância entre avaliadores, em uma etapa subseqüente ${ }^{32}$, foram avaliados 142 pacientes ( 72 agudamente doentes e hospitalizados e 70 cronicamente doentes e residentes em clínicas geriátricas). Os escores modelo 1 (IMC e percentual de perda de peso) e modelo 2 (IMC e albumina sérica) foram empregados por uma técnica em nutrição e uma enfermeira, sendo encontrada concordância $\geq 80,1 \%$ das avaliações, em ambos escores, entre os dois grupos profissionais ( $\kappa=0,6 D P=0,07)$. Quando técnicos de nutrição repetiram a sua própria avaliação, a concordância foi $\geq 70,8 \%(\kappa=0,59 \mathrm{DP}=0,07)$ para o escore nos idosos hospitalizados e $\geq 81,6 \%$ $(\kappa=0,79 \mathrm{DP}=0,06)$ para os residentes em clínicas geriátricas.

\section{Nutritional Screening Tool}

A concordância e a reprodutibilidade do instrumento Nutritional Screening Tool foram descritas por Mackintosh \& Hankey ${ }^{33}$, ao avaliarem 70 idosos no momento da admissão no Ayrshire, Brooksby, Garnock and Maybole Day Hospital, no Reino Unido. Os pacientes foram avaliados e categorizados por enfermeiras quanto a: (a) peso: sem perda de peso (escore $=0$ ), pouca mudança (escore $=1$ ), baixo peso ou sobrepeso (escore $=2$ ) e mudança recente importante (escore $=3$ ), (b) apetite: bom (escore $=0$ ), redução (escore=1), regular (escore $=2$ ) ou pouco ou nenhum (escore $=3)$, (c) ingestão alimentar: $\geq 3$ refeições/ dia (escore $=0$ ), substituição de refeições por lanches (escore $=1$ ), restrição de alimentos ou refeições não concluídas (escore $=2$ ) e falhas freqüente de refeições, precisando ser encorajado a comer (escore=3), (d) ingestão hídrica: $\geq 8$ copos/ dia (escore=0), 6-7 copos $/$ dia (escore=1), 4-5 copos/dia (escore=2) e $\leq 3 \mathrm{copos} / \mathrm{dia}$ (escore=3), (e) habilidade para comer: independente (esco$\mathrm{re}=0$ ), problemas com dentição e demora para comer (escore=1), dificuldade para mastigar, deglutir ou preparar refeições (escore $=2$ ) e dificuldade importante para deglutir, sendo incapaz de alimentar-se sozinho (escore=3), e (f) condições clínicas: não interferem na alimentação (escore=0), infecções repetidas e de curta duração e náuseas (escore $=1$ ), reabilitação gastrintestinal, constipação e tremores (escore=2) e múltiplas doenças, câncer, sepsis, fraturas, úlceras de pressão e tremores acentuados (escore $=3$ ). Pacientes com somatório de escore $\geq 7$ foram referenciados para avaliação adicional por nutricionistas, utilizando IMC e circunferência muscular do braço.

Foi encontrada forte correlação entre os escores de peso $(r=0,94)$, apetite $(r=0,92)$, ingestão alimentar $(r=0,86)$, habilidade para comer $(r=0,90)$, condições clínicas $(r=0,94)$ e ingestão hídrica $(r=0,77)$ entre os 2 grupos de profissionais, assim como concordância em cada categoria avaliada $(\kappa \geq 0,77)$. Foi encontrada correlação negativa entre o NST e prega tricipital, circunferência do braço, circunferência muscular do braço e IMC $(r \geq-0,3)$.

\section{Nutritional Screening Tool}

O Nutritional Screening Too/ ${ }^{\beta 4}$ é um instrumento de triagem nutricional desenvolvido por um grupo de enfermeiras e nutricionistas, testado quanto a sua reprodutibilidade em 100 pacientes, predominantemente mulheres (67\%), internadas por motivos clínicos, cirúrgicos ou geriátricos no Withington Hospital, Reino Unido. Sujeitos que se apresentavam inconscientes, confusos, restritos ao leito ou incapazes de ter o peso aferido foram excluídos do estudo. Trata-se de um questionário, no qual variáveis que influenciam o estado nutricional (idade, nível de consciência, peso, ingestão alimentar, habilidade para alimentar-se, condições clínicas e função intestinal) foram classificadas em 4 categorias, sendo a pontuação $=4$ a de maior comprometimento. 
De acordo com o somatório dos pontos obtidos em cada categoria, os pacientes foram classificados em: risco mínimo (7 a 9 pontos), risco moderado (10 a 14 pontos) e desnutridos ( $\geq 15$ pontos). Nutricionistas também classificaram os pacientes como desnutridos quando IMC $<20 \mathrm{~kg} / \mathrm{m}^{2}$, percentual de perda de peso em 3 meses $>10 \%$, circunferência muscular do braço <percentil $15 \mathrm{e}$ ingestão alimentar $<25 \%$ do recomendado para 24 horas. Estes parâmetros foram adotados como referência para estimativa da acurácia do NST. Os autores encontraram concordância interobservadores de $95 \%$. A NST, quando comparada ao IMC, ao percentual de perda de peso, à circunferência muscular do braço e à ingestão alimentar, apresentou sensibilidade de 59\%, 35\%, 82\% e $59 \%$ e especificidade de $92 \%, 86 \%, 86 \%$ e $86 \%$, respectivamente. Pacientes com escore $\geq 10$ (risco moderado e desnutridos), quando comparados ao diagnóstico de desnutrição por qualquer um dos demais métodos adotados como de referência, apresentaram sensibilidade de $78 \%$, especificidade de $52 \%$, valor preditivo positivo de $74 \%$ e valor preditivo negativo de $57 \%$.

\section{Nutritional Screening Equation}

O estudo que avaliou a Nutritional Screening Equation (NSEq) foi realizado em duas partes: derivação e validação da equação para avaliação do estado nutricional de adultos internados em hospitais de baixa complexidade ${ }^{35}$. A derivação ocorreu por meio de um instrumento com 7 questões que versavam sobre hospitalização prévia, local de moradia, idade, perda de peso, distúrbios alimentares ou de absorção, diagnósticos clínicos e albumina sérica, respondido por 100 pacientes de especialidades de clínica e cirurgia, ou por seus familiares, orientados por voluntários, no momento da admissão hospitalar no St Francis Hospital Center, Indianápolis. Cada variável deveria ser pontuada com valores entre 1 e 6 , conforme a impressão clínica do profissional. Pacientes com somatório de pontos $\geq 6$ deveriam ser encaminhados para avaliação nutricional completa. Os valores de albumina sérica disponíveis nos prontuários dos pacientes também foram revisados $(n=57)$ e pontuados automaticamente com valor $=6$, quando $<35 \mathrm{~g} / \mathrm{L}$. De modo independente, os pacientes foram submetidos a uma avaliação nutricional detalhada que incluiu histórico, exame clínico, medidas bioquímicas e antropométricas, que foi adotada como parâmetro de referência na estimativa de sensibilidade e especificidade. Após procedimento de regressão logística, foi derivada a equação: NSEq=238,664 (albumina) + 0,07242 (contagem total de linfócitos) - 24,657 (percentual de perda de peso). Quando foi avaliado o desempenho isolado do questionário $(n=99)$ foi encontrada sensibilidade de 0,40 e especificidade de 0,95. Ao ser avaliado o desempenho do questionário associado à albumina sérica $(n=58)$, houve aumento da sensibilidade $(0,82)$ e redução da especificidade $(0,65)$, enquanto a NSEq $(n=48)$ mostrou-se sensível $(0,86)$ e específica $(0,96)$. Na segunda etapa, 151 adultos clínicos e cirúrgicos do Community Hospital East, Indianápolis, foram avaliados por técnicos em nutrição, seguindo o mesmo questionário da $1^{\text {a }}$ etapa. Adicionalmente, a pré-albumina dos pacientes foi testada. O questionário, quando isoladamente comparado à avaliação nutricional completa $(n=151)$, mostrou sensibilidade $=0,72$ e especificidade $=0,84$ e combinado à pré-albumina $(n=92)$, a sensibilidade foi 0,61 e a especificidade $=0,91$. A NSEq $(n=151)$ apresentou sensibilidade $=0,93$ e especificidade $=0,85$, tendo redução de sensibilidade $(0,58)$ e aumento de especificidade $(0,97)$, quando associados à pré-albumina $(n=104)$. Os autores sugerem que o custo adicional pela inclusão da pré-albumina à NSEq não está justificado, visto seu pequeno impacto sobre as propriedades preditoras da equação.

\section{CONCLUSÃ O}

A relação causal entre desnutrição e desfechos clínicos hospitalares ainda não é clara, embora piores desfechos clínicos hospitalares 
sejam mais freqüentes em pacientes desnutridos. De fato, a relação temporal entre esses eventos não é suficientemente conhecida, justificando a avaliação do risco nutricional já no momento da admissão hospitalar. No entanto, até o momento, não se dispõe de métodos suficientemente acurados para tal finalidade, dadas as limitações impostas nos procedimentos de derivação e validação dos métodos existentes. Parece inquestionável a necessidade de estabelecer parâmetros de avaliação do estado nutricional de pacientes hospitalizados para que se institua vigilância clínica e epidemiológica, antecipe complicações e, especialmente, adote intervenções precoces. Neste cenário, iniciativas de desenvolvimento e validação de escores são bem-vindas. O melhor método será aquele que contemplar fácil acesso, baixo custo, bom prognóstico e relevância clínica para cada instituição.

\section{A GRADECIMENTOS}

Ao Fundo de Incentivo à Pesquisa e Eventos (FIPE) do Hospital de Clínicas de Porto Alegre e ao Conselho Nacional de Desenvolvimento Científico e Tecnológico (CNPq).

\section{COLABORADORES}

M.G. BEGHETTO participou na concepção e no desenho, na seleção e na revisão da literatura e na redação do artigo. B. MANNA e A. CANDAL participaram na seleção e na revisão da literatura e na redação do artigo. E. D. MELLO e C.A. POLANCZYK participaram na concepção e no desenho, na orientação e na revisão do artigo.

\section{REFERÊ NCIAS}

1. Mello ED, Teixeira LB, Beghetto MG, Luft VC. Desnutrição hospitalar cinco anos após o IBRANUTRI. Rev Bras Nutr Clin. 2003; 18(2):65-9.

2. Waitzberg DL, Caiaffa WT, Correia MI. Hospital malnutrition: the Brazilian national survey (IBRANUTRI): a study of 4000 patients. Nutrition. 2001: 17(7-8):573-80.
3. Naber TH, Schermer T, Bree A, Nusteling K, Eggink $\mathrm{L}$, Kruimel JW, et al. Prevalence of malnutrition in nonsurgical hospitalized patients and its association with disease complications. Am J Clin Nutr. 1997; 66(5):1232-9.

4. Symreng T, Anderberg B, Kagedal B, Norr A, Schildt B, Sjodahl R. Nutritional assessment and clinical course in 112 elective surgical patients. Acta Chir Scand. 1983; 149(7):657-62.

5. Correia Ml, Campos AC, ELAN Cooperative Study. Prevalence of hospital malnutrition in Latin America: the multicenter ELAN study. Nutrition. 2003; 19(10):823-5.

6. Edington J, Boorman J, Durrant ER, Perkins A, Giffin $C V$, James $R$, et al. Prevalence of malnutrition on admission to four hospitals in England. Clin Nutr. 2000; 19(3):191-5.

7. Reilly JJ Jr, Hull SF, Albert N, Waller A, Bringardener S. Economic impact of malnutrition: a model system for hospitalized patients. JPEN J Parenter Enteral Nutr. 1988; 12(4):371-6.

8. Larsson J, Akerlind I, Permerth J, Hornqvist JO. The relation between nutritional state and quality of life in surgical patients. Eur J Surg. 1994; 160(6-7): 329-34.

9. Akner G, Cederholm T. Treatment of proteinenergy malnutrition in chronic nonmalignant disorders. Am J Clin Nutr. 2001; 74(1):6-24.

10. Boog MCF, Silva JB. Percepções de enfermeiras sobre o processo de cuidado nutricional. Rev Bras Nutr Clin. 2001; 16(1):17-22.

11. Roubenoff R, Roubenoff RA, Preto J, Balke CW. Malnutrition among hospitalized patients. A problem of physician awareness. Arch Intern Med. 1987; 147(8):1462-5.

12. Kondrup J, Allison SP, Elia M, Vellas B, Plauth M. ESPEN guidelines for nutrition screening 2002. Clin Nutr. 2003; 22(4):415-21

13. Identifying patients at risk: ADA's definitions for nutrition screening and nutrition assessment. Council on Practice (COP) Quality Management Committee. J Am Diet Assoc. 1994; 94(8):838-89.

14. Barrocas A, Belcher D, Champagne C, Jastram C. Nutrition assessment practical approaches. Clin Geriatr Med. 1995; 11(4):675-713.

15. Ferguson M, Capra S, Bauer J, Banks M. Development of a valid and reliable malnutrition screening tool for adult acute hospital patients. Nutrition. 1999; 15(6):458-64.

16. Kruizenga HM, Seidell JC, Vet HC, Wierdsma NJ, van Bokhorst-de van der Schueren MA Development and validation of a hospital screening tool for malnutrition: the short nutritional 
assessment questionnaire (SNAQ). Clin Nutr. 2005; 24(1):75-82.

17. Wolinsky FD, Coe RM, Chavez MN, Prendergast JM, Miller DK. Further assessment of the reliability and validity of a Nutritional Risk Index: analysis of a three-wave panel study of elderly adults. Health Serv Res. 1986; 20(6 Pt 2):977-90.

18. Buzby GP, Williford WO, Peterson OL, Crosby LO, Page CP, Reinhardt GF, et al. A randomized clinical trial of total parenteral nutrition in malnourished surgical patients: the rationale and impact of previous clinical trials and pilot study on protocol design. Am J Clin Nutr. 1988; 47(Suppl 2):357-65.

19. Prendergast JM, Coe RM, Chavez MN, Romeis JC, Miller DK, Wolinsky FD. Clinical validation of a nutritional risk index. J Comm Health. 1989; 14(3): 125-35.

20. Schneider SM, Veyres P, Pivot $X$, Soummer AM, Jambou P, Filippi J, et al. Malnutrition is an independent factor associated with nosocomial infections. Br J Nutr. 2004; 92(1):105-11.

21. Kyle UG, Schneider SM, Pirlich M, Lochs $H$, Hebuterne $X$, Pichard C. Does nutritional risk, as assessed by Nutritional Risk Index, increase during hospital stay? A multinational population-based study. Clin Nutr. 2005; 24(4):516-24.

22. The Veterans Affairs Total Parenteral Nutrition Cooperative Study Group. Perioperative total parenteral nutrition in surgical patients. $N$ Engl J Med. 1991; 325(8):525-32.

23. Reilly HM, Martineau JK, Moran A, Kennedy H. Nutritional screening: evaluation and implementation of a simple Nutrition Risk Score. Clin Nutr. 1995; 14(5):269-73.

24. Wolinsky FD, Coe RM, McIntosh W, Kubena KS, Prendergast JM, Chavez MN, et al. Progress in the development of a Nutritional Risk Index. J Nutr. 1990; 120(Suppl 11):1549-53.

25. Corish CA, Flood P, Kennedy NP. Comparison of nutritional risk screening tools in patients on admission to hospital. J Hum Nutr Diet. 2004; 17(2):133-9.

26. Kondrup J, Rasmussen HH, Hamberg O, Stanga Z, Ad Hoc ESPEN Working Group. Nutritional risk screening (NRS 2002): a new method based on an analysis of controlled clinical trials. Clin Nutr. 2003; 22(3):321-36.

27. Guigoz Y, Vellas B, Garry PJ. Mini nutritional assessment: a practical assessment tool for grading the nutritional state of ederly patients. Facts Res Gerontol. 1994; 1(Suppl 2):S15-S59.

28. Guigoz Y, Vellas B, Garry PJ. Assessing the nutritional status of the elderly: the Mini Nutritional Assessment as part of the geriatric evaluation. Nutr Rev. 1996; 54(1 Pt 2):S59-65.

29. Van Nes MC, Herrmann FR, Gold G, Michel JP, Rizzoli R. Does the Mini Nutritional Assessment predict hospitalization outcomes in older people? Age Ageing. 2001; 30(3):221-6.

30. Stratton RJ, Hackston A, Longmore D, Dixon R, Price S, Stroud M, et al. Malnutrition in hospital outpatients and inpatients: prevalence, concurrent validity and ease of use of the 'malnutrition universal screening tool' ('MUST') for adults. Br J Nutr. 2004; 92(5):799-808.

31. Laporte M, Villalon L, Payette H. Simple nutrition screening tools for healthcare facilities: development and validity assessment. Can J Diet Pract Res. 2001; 62(1):26-34.

32. Laporte M, Villalon L, Thibodeau J, Payette H. Validity and reliability of simple nutrition screening tools adapted to the elderly population in healthcare facilities. J Nutr Health Aging. 2001; 5(4):292-4.

33. Mackintosh MA, Hankey CR. Reliability of a nutrition screening tool for use in elderly day hospitals. J Hum Nutr Diet. 2001; 14(2):129-36.

34. Burden ST, Bodey S, Bradburn YJ, Murdoch S, Thompson AL, Sim JM et al. Validation of a nutrition screening tool: testing the reliability and validity. J Hum Nutr Diet. 2001; 14(4):269-75.

35. Elmore MF, Wagner DR, Knoll DM, Eizember L, Oswalt MA, Glowinski EA et al. Developing an effective adult nutrition screening tool for a community hospital. J Am Diet Assoc. 1994; 94(10):1113-8.

Recebido em: 26/10/2006

Versão final reapresentada em: 6/12/2007 Aprovado em: 13/5/2008 